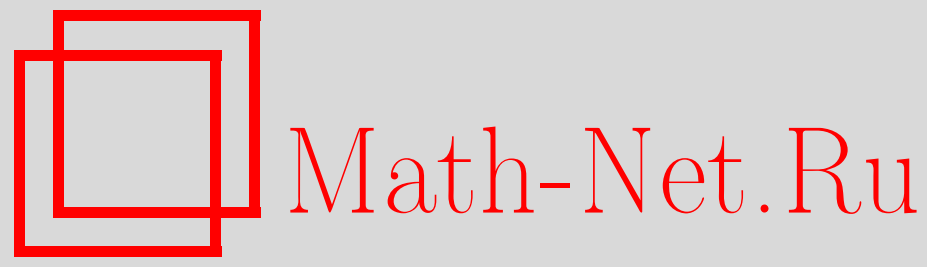

Г. С. Мустафаев, О радикале некоторого класса банаховых алгебр, Функи. анализ и его прил., 2007, том 41, выпуск 3, 89-93

DOI: https://doi.org/10.4213/faa2870

Использование Общероссийского математического портала MathNet.Ru подразумевает, что вы прочитали и согласны с пользовательским соглашением

http://www.mathnet.ru/rus/agreement

Параметры загрузки:

IP : 3.80 .253 .173

26 апреля 2023 г., 18:13:56

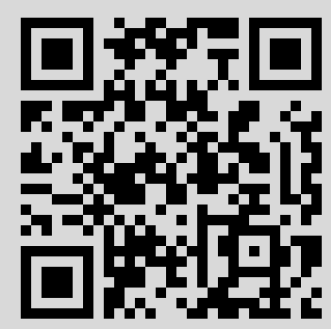




\title{
О радикале некоторого класса банаховых алгебр
}

\author{
(C) 2007. Г. С. Мустафаев
}

Пусть $A$ - комплексная банахова алгебра. Известно ([1, с. 50], [2], [3]), что во втором сопряженном пространстве $A^{* *}$ можно определить умножение (относительно которого $A^{* *}$ становится банаховой алгеброй), являющееся естественным продолжением исходного умножения в $A$. Это умножение (которое называется умножением Аренса), определяется следующим образом:

(a) $\langle\varphi \cdot a, b\rangle=\langle\varphi, a b\rangle$ для всех $a, b \in A$ и $\varphi \in A^{*}$;

(b) $\langle H \cdot \varphi, a\rangle=\langle H, \varphi \cdot a\rangle$ для всех $a \in A, \varphi \in A^{*}$ и $H \in A^{* *}$;

(c) $\langle F \circ H, \varphi\rangle=\langle F, H \cdot \varphi\rangle$ для всех $\varphi \in A^{*}$ и $F, H \in A^{* *}$.

Алгебру $A$ можно считать подалгеброй алгебры $A^{* *} . \operatorname{Kak~обычно,~} \operatorname{Rad}(A)$ будет обозначать радикал (Джекобсона) алгебры $A$. Цивин и Юд $[2$, теорема 3.15] доказали, что если $G$ - компактная абелева группа, то

$$
\operatorname{Rad}\left(L^{1}(G)^{* *}\right)=C(G)^{\perp}=\left(L^{\infty}(G) * L^{1}(G)\right)^{\perp} .
$$

В этой заметке мы покажем, что для некоторых классов банаховых алгебр $A$ с вполне несовершенным структурным пространством справедливы равенства $\operatorname{Rad}(A)={ }^{\perp}\left(A^{*} \cdot A\right)$ и $\operatorname{Rad}\left(A^{* *}\right)=\left(A^{*} \cdot A\right)^{\perp}$, где

$$
A^{*} \cdot A=\left\{\varphi \cdot a: \varphi \in A^{*}, a \in A\right\} .
$$

Напомним, что замкнутое подмножество $S$ хаусдорфова топологического пространства называется вполне несовершенным, если оно не содержит непустых совершенных подмножеств. Через $\widehat{A}$ будем обозначать множество классов эквивалентности неприводимых представлений алгебры $A$.

Лемма 1. Пусть $A$ - банахова алгебра, такая, что любое ее неприводимое представление конечномерно. Тогда справедливы следующие утверждения:

(а) если $A$ имеет ограниченную аппроксимативную единииу (о.а.е.), то

$$
\overline{\operatorname{span}}\left\{(\operatorname{ker} \sigma)^{\perp}: \sigma \in \widehat{A}\right\} \subset A^{*} \cdot A ;
$$

(b) если $A^{*} \cdot A \subset \overline{\operatorname{span}}\left\{(\operatorname{ker} \sigma)^{\perp}: \sigma \in \widehat{A}\right\}$, mo

$$
\operatorname{Rad}(A)={ }^{\perp}\left(A^{*} \cdot A\right) \quad \text { u } \quad \operatorname{Rad}\left(A^{* *}\right)=\left(A^{*} \cdot A\right)^{\perp} .
$$

На основе этой леммы мы получаем такую теорему:

Теорема 2. Пусть A - полупростал банахова алгебра, такал, что любое ее неприводимое представление конечномерно. Если операторы умножения слева (соответственно справа) в А на элементы из А слабо компактны, то

$$
\operatorname{Rad}\left(A^{* *}\right)=\left(A^{*} \cdot A\right)^{\perp} .
$$

Если $G$ - компактная группа, то алгебра $L^{1}(G)$ полупроста и любое ее неприводимое представление конечномерно. С другой стороны, из теории ПетераВейля следует, что в $L^{1}(G)$ полна система конечномерных левых (соответственно правых) идеалов. Отсюда вытекает, что для произвольного $f \in L^{1}(G)$ оператор умножения слева $L_{f}$ (соответственно оператор умножения справа $R_{f}$ ) 
аппроксимируется (по операторной норме) конечномерными операторами. Следовательно, $L_{f}$ (соответственно $\left.R_{f}\right)$ - компактный оператор. Далее, поскольку

$$
L^{\infty}(G) \cdot L^{1}(G)=L^{\infty}(G) * L^{1}(G)=C(G),
$$

то из теоремы 2 получаем такое

Следствие 3. Если $G$ - компактная группа (не обязательно абелева), то

$$
\operatorname{Rad}\left(L^{1}(G)^{* *}\right)=C(G)^{\perp} .
$$

Пусть $A$ - банахова алгебра с о.а.е. и $E$ есть $\sigma\left(A^{* *}, A^{*}\right)$-предельная точка о.а.е. в $A$. Нетрудно заметить, что $F \circ E=F$ при всех $F \in A^{* *}$. Заметим также, что отображение $P_{E}: A^{* *} \rightarrow A^{* *}$, определенное равенством $P_{E}(F)=E \circ F$, является ограниченным проектором и поэтому индуцирует разложение

$$
A^{* *}=P_{E}\left(A^{* *}\right) \oplus\left(1-P_{E}\right) A^{* *} \text {. }
$$

Нетрудно заметить, что $P_{E}\left(A^{* *}\right)$ является замкнутой подалгеброй алгебры $A^{* *}$ и $\left(1-P_{E}\right) A^{* *}=\left(A^{*} \cdot A\right)^{\perp}$.

Говорят [1, с. 253], что банахова алгебра $A$ допускает сильное веддербёрновское разложсние, если существует такая замкнутая подалгебра $B$ алгебры $A$, что $A=B \oplus \operatorname{Rad}(A)$.

Следствие 4. Пусть $A$ - полупростая банахова алгебра, такая, что любое ее неприводимое представление конечномерно. Предположим, что $А$ имеет о.а.е. и операторы умножения слева (соответственно справа) в А на элементы из $A$ слабо компактны. Тогда $A^{* *}$ допускает силъное веддербёрновское разложсение.

Пусть $A$ - коммутативная банахова алгебра, а $M_{A}$ - ее пространство максимальных идеалов. Через $\hat{a}$ будем обозначать преобразование Гельфанда элемента $a \in A$. Если $A$ не имеет единичного элемента, то алгебру, полученную из $A$ присоединением единицы, будем обозначать через $A_{1}$.

Пусть $A$ - регулярная (по Шилову) полупростая банахова алгебра и $A_{00}=$ $\{a \in A: \operatorname{supp} \hat{a}$ компактен $\}$. Хорошо известно, что $J_{\phi}=\overline{\left\{a \in A_{00}: \phi \notin \operatorname{supp} \hat{a}\right\}}$ для произвольного $\phi \in M_{A}$ есть наименьший замкнутый идеал в $A$ с оболочкой $\{\phi\}$, а $I_{\phi}=\{a \in A: \hat{a}(\phi)=\{0\}\}$ - наибольший замкнутый идеал с оболочкой $\{\phi\}$. Алгебра $A$ называется сильно регулярной, если каждая точка из $M_{A}$ (а в случае, когда $A$ не имеет единичного элемента, то $\{\infty\}$ ) является множеством синтеза для $A$ (для $A_{1}$ ). Таким образом, $A$ сильно регулярна тогда и только тогда, когда $I_{\phi}=J_{\phi}$ для всех $\phi \in M_{A}$ и $\overline{A_{00}}=A$. Напомним также, что $w^{*}$-спектр $\sigma_{*}(\varphi)$ функционала $\varphi \in A^{*}$ определяется как оболочка идеала

$$
I_{\varphi}=\{a \in A: \varphi \cdot a=0\} .
$$

Теорема 5. Пусть $A$ - сильно регулярная банахова алгебра, а $B$ - коммутативная банахова алгебра, такая, что $M_{B}$ дискретно. Если существует непрерывный гомоморфизм $h: A \rightarrow B$ с плотным образом, то

$$
\operatorname{Rad}(B)={ }^{\perp}\left(B^{*} \cdot B\right) \quad \text { u } \operatorname{Rad}\left(B^{* *}\right)=\left(B^{*} \cdot B\right)^{\perp} .
$$

Напомним [9, с. 13], что если $A$ - коммутативная банахова алгебра, то $\perp\left(A^{*} \cdot A\right)$ называется ее аннуляторным идеалом. 
Следствие 6. Пусть $A-$ сильно регулярная банахова алгебра, а $B$ - коммутативная банахова алгебра, такая, что $M_{B}$ дискретно. Предположим, что существует непрерывный гомоморфизм $h: A \rightarrow B$ с плотным образом. Тогда В является полупростой только в том случае, когда она имеет нулевой аннуляторный идеал.

Следующий пример показывает, что без условия сильной регулярности следствие 6 перестает быть верным.

Пример 7. Пусть $A=C^{1}[-1,1]$ - банахова алгебра непрерывно дифференцируемых функций на $[-1,1]$. Тогда $I_{0}=\{f \in A: f(0)=0\}$ и $J_{0}=\{f \in$ $\left.A: f(0)=f^{\prime}(0)=0\right\}[5, \S 37]$. Поэтому $A$ не является сильно регулярной. Если положить $B=A / J_{0}$, то можно убедиться, что $B$ имеет нулевой аннуляторный идеал и $M_{B}=\{0\}$. Однако $\operatorname{Rad}(B)=I_{0} / J_{0} \neq\{0\}$.

Пусть $X$ - банахово пространство, $B(X)$ - алгебра ограниченных линейных операторов в $X$ и $T \in B(X)^{-1}$. Известная теорема Гельфанда [4] утверждает, что если $\sup _{n \in \mathbb{Z}}\left\|T^{n}\right\|<\infty$ и $\sigma(T)=\{1\}$, то $T=I$ - единичный оператор в $X$. В качестве обобщения теоремы Гельфанда в монографии Хилле и Филлипса [8, теорема 4.10.1] доказано, что если $T \in B(X)^{-1}$, причем $\left\|T^{n}\right\|=o(|n|)$ и $\sigma(T)=\{1\}$, то $T=I$.

Как применение теоремы 5 ниже приводится более общий результат.

Пусть $G$ - локально компактная абелева группа, а $\widehat{G}$ - группа ее характеров. Напомним, что весовая функиия - это непрерывная функция $\omega(g)$ на $G$ со свойствами $\omega(g) \geqslant 1$ и $\omega(g+s) \leqslant \omega(g)+\omega(s)$ для всех $g, s \in G$. Алгебра Берлинга $c$ весом $\omega$ задается формулой

$$
L_{\omega}^{1}(G)=\left\{f \in L^{1}(G):\|f\|_{\omega}=\int_{G}|f(g)| \omega(g) d g<\infty\right\} .
$$

Известно [15, с. 132], что если $\omega-$ весовая функция на $G$, удовлетворяющая условию Шилова, то алгебра $L_{\omega}^{1}(G)$ сильно регулярна.

Пусть $g \rightarrow T(g)$ - равномерно непрерывное представление группы $G$ в банаховом пространстве $X$. Заметим, что функция

$$
\omega_{T}(g)=\max \{\|T(g)\|,\|T(-g)\|\}
$$

является весовой функцией на $G$. Если положить $L_{T}(G)=\overline{\operatorname{span}}\{T(g): g \in G\}$, то $L_{T}(G)$ - коммутативная банахова алгебра с единицей $I$. Для произвольной функции $f \in L_{\omega_{T}}^{1}(G)$ определим $T_{f} \in B(X)$ равенством $T_{f}=\int_{G} f(g) T(g) d g$. Нетрудно проверить, что отображение $h: L_{\omega_{T}}^{1}(G) \rightarrow L_{T}(G)$, определяемое равенством $h(f)=T_{f}$, является непрерывным гомоморфизмом с плотным образом. В силу следствие 6 получаем, что если 1$)$ весовая функция $\omega_{T}(g)$ удовлетворяет условию Шилова и 2) пространство максимальных идеалов алгебры $L_{T}(G)$ - конечное множество, то $L_{T}(G)$ - полупростая алгебра.

Теперь, применяя теорему Шилова об идемпотентах из [1, с. 109], получаем такое

Следствие 8. Предположим, что пространство максимальных идеалов алгебры $L_{T}(G)$ состоит из $n$ точек и весовая функиия $\omega_{T}(g)$ удовлетворяет следующим условиям Шилова:

(i) $\omega_{T}(n g)=O\left(|n|^{\alpha}\right)$ для некоторого $\alpha=\alpha(g)>0$ и для каждого $g \in G$ при $|n| \rightarrow \infty$; 
(ii) $\lim _{|n| \rightarrow \infty} \inf \frac{\omega_{T}(n g)}{|n|}=0$ для каждого $g \in G$.

Тогда существуют попарно ортогональные идемпотенты $p_{1}, \ldots, p_{n}$ в $L_{T}(G)$, такие, что $p_{1}+\cdots+p_{n}=I$ и $T(g)=\chi_{1}(g) p_{1}+\cdots+\chi_{n}(g) p_{n}$ для некоторьх $\chi_{1}, \ldots, \chi_{n}$ в $\widehat{G}$.

Следующий результат является частным случаем одной теоремы Горина [6].

Теорема 9. Пусть $A-$ - коммутативная банахова алгебра с вполне несовершенным пространством максимальных идеалов. Если существует непрерывный гомоморфизм $h: L^{1}(G) \rightarrow A$ с плотным образом, то $A$ - полупростая алгебра.

Заметим, что предположений теоремы 9 недостаточно для полупростоты алгебры $A^{* *}$. Чтобы убедиться в этом, положим $A=L^{1}(G)$, где $G$ - компактная абелева группа. Так как $M_{A}(=\widehat{G})$ дискретно, то оно вполне несовершенно. Но алгебра $A^{* *}$ не является полупростой, потому что в силу теоремы Цивина и Юда, приведенной выше, $\operatorname{Rad}\left(A^{* *}\right)=C(G)^{\perp}$.

Однако имеет место следующая

Теорема 10. Пусть $A-$ коммутативнал банахова алгебра с вполне несовершенным пространством максимальных идеалов, и пусть существует непрерывный гомоморфизм $h: L^{1}(G) \rightarrow A$ с плотным образом. Если алгебра $A^{* *}$ коммутативна, то она полупростая.

Известная теорема Люмиса [12] утверждает, что если $w^{*}$-спектр функции $\varphi \in$ $L^{\infty}(G)$ компактен и вполне несовершенен, то $\varphi$ является почти периодической, а именно $\varphi \in \overline{\operatorname{span}} \sigma_{*}(\varphi)$. С помощью теоремы Люмиса мы получаем следующее обобщение теоремы Цивина и Юда.

Теорема 11. Пусть $I$ - замкнутый идеал в $L^{1}(G)$ с вполне несовершенной оболочкой и $A=L^{1}(G) / I$. Тогда

$$
\operatorname{Rad}\left(A^{* *}\right)=\left(A^{*} \cdot A\right)^{\perp} .
$$

С помощью векторного варианта теоремы Люмиса $[11, \S 4$, теорема 4] получаем такую теорему:

Теорема 12. Пусть $A$ - коммутативная банахова алгебра с вполне несовершенным пространством максимальных идеалов, и пусть существует непрерывный гомоморфизм $h: L^{1}(G) \rightarrow A$ с плотным образом. Если $c_{0} \not \subset A^{*}$, то

$$
\operatorname{Rad}\left(A^{* *}\right)=\left(A^{*} \cdot A\right)^{\perp} \text {. }
$$

\section{ЛитеРАтУРА}

[1] F. F. Bonsall, J. Duncan, Complete Normed Algebras, Springer-Verlag, New YorkHeidelberg, 1973. [2] P. Civin, B. Yood, Pacific J. Math., 11 (1961), 847-870. [3] J. Duncan, S. A. R. Hosseiniun, Proc. Roy. Soc. Edinburgh Sect. A, 84:3-4 (1979), 309-325. [4] И. Гельфанд, Матем. сб., 9 (1941), 49-50. [5] I. Gelfand, D. Raikov, G. Shilov, Commutative Normed Rings, Chelsea Publ. Comp., 1964 (расширенный перевод книги И. М. Гельфанда, Д. А. Райкова, Г. Е. Шилова «Коммутативные нормированные кольца», Физматлит, М., 1960). [6] Е. А. Горин, Функц. анализ и его прил., 8:2 (1974), 73-74. [7] Хьюитт Э., Росс К., Абстрактный гармонический анализ, т. 2, Наука, М., 1975. [8] Э. Хилле, Р. Филлипс, Функциональный анализ и полугруппы, ИЛ, М., 1962. [9] R. Larsen, An Introduction to the Theory of Multipliers, Springer-Verlag, New York-Heidelberg, 1971. [10] R. Larsen, Banach Algebras, Marcel Dekker, New York, 1973. 
[11] Б. М. Левитан, В. В. Жиков, Почти периодические функиии и дифференциальные уравнения, Изд-во МГУ, М., 1978. [12] L. H. Loomis, Ann. Math. (2), 72 (1960), 362-368. [13] Ю. И. Любич, Введение в теорию банаховых представлений групп, Высшая школа, Харьков, 1985. [14] H. S. Mustafayev, Bull. Polish. Acad. Sci. Math., 52:4 (2004), 395-403. [15] H. Reiter, Classical Harmonic Analysis and Locally Compact Groups, Clarendon Press, Oxford, 1968. [16] C. Rickart, General Theory of Banach Algebras, Van Nostrand, Princeton, 1960.

Факультет естественных и гуманитарных наук Университета Йузунджу Йыл, Турция

Поступило в редакцию e-mail: hsmustafayev@yahoo.com

28 июня 2005 г.

УДК 517.983.243, 517.984.4

\title{
О сходимости функций нормальных операторов в сильной операторной топологии*
}

\author{
(c) 2007. О. Е. Тихонов
}

Всюду ниже $\mathscr{N}(H)$ обозначает множество всех ограниченных нормальных операторов в гильбертовом пространстве $H$, а $\operatorname{Sp}(A)$ - спектр оператора $A$. Для числовой функции $f$ соответствующая ей по спектральной теореме операторная функция обозначается той же буквой. Символ $\stackrel{s}{\rightarrow}$ используется для обозначения сходимости в сильной операторной топологии (далее - в $s$-топологии).

В работе Дэвиса [1] доказана следующая

Теорема. Пусть $A, A_{n} \in \mathscr{N}(H)$, и пусть $A_{n} \stackrel{s}{\rightarrow}$ A. Пусть $f-$ ограниченная борелевская функиия, определенная на $\mathbb{C}, u \Gamma-$ замкнутое подмножество в $\operatorname{Sp}(A)$, спектральный проектор которого относительно А равен нулю. Пусть $f$ непрерьвна в каждой точке из $\mathrm{Sp}(A) \backslash \Gamma$. Тогда $f\left(A_{n}\right) \stackrel{s}{\rightarrow} f(A)$.

Цель настоящей заметки - обобщить эту теорему таким образом, чтобы получить уточнение теоремы Кэдисона [2] о сильной непрерывности операторных функций на подмножествах нормальных операторов. При доказательстве применяются результаты и конструкции работ [1], [2]. Существенно новый момент - использование следующей топологической леммы.

Лемма 1. Пусть $(X, d)$ - такое метрическое пространство, что все его

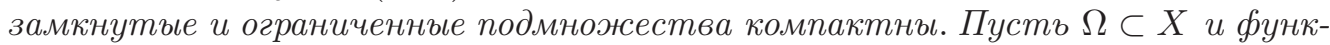
иия $f: \Omega \rightarrow[a, b]$ непрерьвна на $\Omega$. Тогда существует функиия $g: X \rightarrow[a, b]$, совпадающая с $f$ на $\Omega$, непрерьвная во всех точках из $\Omega$ и полунепрерывная сверху на $X$.

Доказательство. Для произвольной точки $x^{\prime}$ из замыкания $\bar{\Omega}$ множества $\Omega$ положим $g_{1}\left(x^{\prime}\right)=\lim \sup _{x \in \Omega, x \rightarrow x^{\prime}} f(x)$. Через $\operatorname{dist}(x, \Delta)$ обозначим расстояние между точкой $x \in X$ и множеством $\Delta \subset X$. Для произвольного $x \in X$ рассмотрим непустое компактное множество $\Omega_{x}=\left\{x^{\prime} \in \bar{\Omega} \mid d\left(x, x^{\prime}\right)=\operatorname{dist}(x, \bar{\Omega})\right\}$ и положим $g(x)=\sup \left\{g_{1}\left(x^{\prime}\right) \mid x^{\prime} \in \Omega_{x}\right\}$. Стандартным образом проверяется, что функция $g$ удовлетворяет всем требуемым условиям.

*Работа поддержана РФФИ, проект 05-01-00799. 